Then, for $G \simeq K$ it is necessary and sufficient that $R$ be cyclically equivalent to $A^{n}\left(a_{1}, a_{2}\right)$ where $A\left(a_{1}, a_{2}\right)$ is a primitive element in the free group $F\left(a_{1}, a_{2}\right)$.

\title{
REFERENCES
}

1. J. H. C. Whitehead, On equivalent sets of elements in a free group, Ann. of Math. vol. 37 (1936).

2. A. G. Kurosh, Theory of groups, Gostekhizdat, 1944 (in Russian).

NEW YoRK UNIVERSITY

\section{A THEOREM ON COMMUTATIVE POWER ASSOCIATIVE LOOP ALGEBRAS ${ }^{1}$}

\section{LOWELl J. PAIGE}

Let $L$ be a loop, written multiplicatively, and $F$ an arbitrary field. Define multiplication in the vector space $A$, of all formal sums of a finite number of elements in $L$ with coefficients in $F$, by the use of both distributive laws and the definition of multiplication in $L$. The resulting loop algebra $A(L)$ over $F$ is a linear nonassociative algebra (associative, if and only if $L$ is a group).

An algebra $A$ is said to be power associative if the subalgebra $F[x]$ generated by an element $x$ is an associative algebra for every $x$ of $A$.

Theorem. Let $A(L)$ be a loop algebra over a field of characteristic not 2. A necessary and sufficient condition that $A(L)$ be a commutative, power associative algebra is that $L$ be a commutative group.

Proof. Assume that $A(L)$ is a commutative, power associative algebra. Clearly $L$ must be commutative and $x^{2} \cdot x^{2}=\left(x^{2} \cdot x\right) \cdot x$ for all $x$ of $A(L)$. Under the hypothesis that the characteristic of $F$ is not 2 , a linearization ${ }^{2}$ of this power identity yields

Presented to the Society, December 28, 1953; received by the editors June 2, 1954.

1 The preparation of this paper was sponsored in part by the Office of Naval Research.

2 See A. A. Albert, On the power associativity of rings, Summa Brasiliensis Mathematicae vol. 2 , no. 2 , pp. 21-32. 


$$
\sum_{6}(x y+y x)(z w+w z)=\sum_{4}\left[\sum_{3}(z w+w z) y\right] x,
$$

for all $x, y, z, w$ of $A(L)$. The sums in (1) are taken over all possible selections of the symbols involved and $\sum_{k}$ is the sum of $k$ terms. We now restrict our attention to elements of $L$ in $A(L)$ and set $w=z$ in (1). Thus we obtain,

$$
\begin{array}{r}
4 z^{2}(x y)+8(x z \cdot z y)=\left(z^{2} y\right) x+\left(z^{2} x\right) y+2(y z \cdot z) x+2(x z \cdot z) y \\
+2(z y \cdot x) z+2(z x \cdot y) z+2(x y \cdot z) z
\end{array}
$$

as a necessary condition for the power associativity of $A(L)$.

The left member of (2) consists of at most two distinct elements of $L$. Hence the resolution of the right member into two distinct elements of $L$ requires that two of the three elements $(z y \cdot x) z,(z x \cdot y) z$, $(z y \cdot z) z$ be equal. This, together with the commutativity of $L$, implies that $(x y) z=x(y z)$ for all $x, y, z$ of $L$.

The proof of the sufficiency is obvious.

The situation is expectedly different if the characteristic of $F$ is two. For example, let $L$ be a commutative loop such that $x^{2}=1$ ( 1 the identity element of $L$ ) for all $x$ of $L$. Then if $\alpha=\sum_{i=1}^{n} a_{i} x_{i}$, where $a_{i} \in F$ and $x_{1}=1$,

$$
\alpha^{2}=\left[\sum_{i=1}^{n} a_{i}^{2}\right] x_{1}
$$

hence, the power associativity of the loop algebra $A(L)$ is a trivial verification.

University of California, Los Angeles and

The Institute for Advanced Study 